International Journal of Applied Mathematics

Volume 34 No. $4 \quad 2021,653-668$

ISSN: 1311-1728 (printed version); ISSN: 1314-8060 (on-line version)

doi: http://dx.doi.org/10.12732/ijam.v34i4.5

\title{
SINGULARLY PERTURBED INTEGRAL EQUATIONS WITH A RAPIDLY OSCILLATING INHOMOGENEITY
}

\author{
Burkhan Kalimbetov $^{1}$, Valeriy Safonov ${ }^{2}$, Elvira Madikhan ${ }^{1}$ \\ ${ }^{1}$ Khoja Ahmet Yasawi International \\ Kazakh-Turkish University \\ Department of Mathematics \\ B. Sattarkhanov 29, Turkestan \\ 161200, KAZAKHSTAN \\ ${ }^{2}$ National Research University, MPEI \\ Department of Higher Mathematics \\ Krasnokazarmennaya 14, Moscow \\ 111250, RUSSIA
}

\begin{abstract}
In this paper, we consider singularly perturbed integro-differential equations with a rapidly oscillating right-hand side, including an integral operator with a slowly varying kernel. The main goal of this work is to generalize the Lomov's regularization method and to reveal the influence of the rapidly oscillating right-hand side on the asymptotics of the solution to the original problem.
\end{abstract}

AMS Subject Classification: 35R09, 45K05

Key Words: singular perturbation; integro-differential equation; regularization; rapidly oscillating inhomogeneity; asymptotic convergence

\section{Introduction}

When studying various applied problems related to the properties of media with a period structure, it is necessary to study differential equations with rapidly oscillating irregularities. Equations of this type are often found, for example, in electrical tric systems under the influence of high-frequencyexternal

Received: January 29, $2021 \quad$ (C) 2021 Academic Publications

${ }^{\S}$ Correspondence author 
forces. The presence of such forces creates serious problems for the numerical integration of the corresponding differential equations. Therefore, asymptotic methods are usually applied to such equations, the most famous of which are the Feshchenko - Shkil - Nikolenko splitting method [1,2] and the Lomov's regularization method, [3-13]. Splitting method especially is effective when applied to equations with a rapidly oscillating inhomogeneity, and in the case of an inhomogeneity containing both rapidly and slow components, the Lomov's regularization method turned out to be the most effective. However, both of these methods were developed mainly for singularly perturbed equations that do not contain an integral operator. The transition from differential equations to integro-differential equations requires a significant restructuring of the algorithm of the regularization method. The integral term generates new types of singularities in solutions that differ from the previously known ones, which complicates the development of the algorithm for the regularization method. As far as we know, the splitting method has not been applied to integro-differential equations.

\section{Statement of the problem and reduction of the equation (1) to an integro-differential problem}

Consider the singularly perturbed integral equation

$$
\begin{aligned}
& L_{\varepsilon} y(t, \varepsilon) \equiv \varepsilon y-\int_{0}^{t} K(t, s) y(s, \varepsilon) d s \\
& =h_{1}(t)+\varepsilon h_{2}(t) e^{\frac{i \beta(t)}{\varepsilon}}, t \in[0, T],
\end{aligned}
$$

where $\varepsilon>0$ is a small parameter, $h_{1}(t), h_{2}(t), K(t, s)$ are known functions, $y(t)$ is unknown function, and $\beta^{\prime}(t)>0(\forall t \in[0, T])$ is known scalar function. In the development of an algorithm for constructing an asymptotic solution of the problem (1), an important role is played by essentially singularities in the solution of this equation. To describe them, we differentiate equation (1) by $t$; will have

$$
\begin{gathered}
L_{\varepsilon} y(t, \varepsilon) \equiv \varepsilon y^{\prime}-K(t, t) y-\int_{0}^{t} \frac{\partial K(t, s)}{\partial t} y(s, \varepsilon) d s \\
=h_{1}^{\prime}(t)+\varepsilon h_{2}^{\prime}(t) e^{\frac{i \beta(t)}{\varepsilon}}+i \beta^{\prime}(t) h_{2}(t) e^{\frac{i \beta(t)}{\varepsilon}}, \\
y(0, \varepsilon)=\frac{h_{1}(0)}{\varepsilon}+h_{2}(0) e^{\frac{i \beta(0)}{\varepsilon}} .
\end{gathered}
$$


Let us denote by $\lambda_{1}(t) \equiv K(t, t), G(t, s) \equiv \frac{\partial K(t, t)}{\partial t}$ and by $\beta^{\prime}(t)$ the frequency of a rapidly oscillating inhomogeneity. Then problem (2) can be rewritten as follows:

$$
\begin{aligned}
& L_{\varepsilon} y(t, \varepsilon) \equiv \varepsilon y^{\prime}-\lambda_{1}(t) y-\int_{0}^{t} G(t, s) y(s, \varepsilon) d s \\
& =h_{1}^{\prime}(t)+\varepsilon h_{2}^{\prime}(t) e^{\frac{i \beta(t)}{\varepsilon}}+i \beta^{\prime}(t) h_{2}(t) e^{\frac{i \beta(t)}{\varepsilon}}, \\
& y(0, \varepsilon)=\frac{h_{1}(0)}{\varepsilon}+h_{2}(0) e^{\frac{i \beta(0)}{\varepsilon}}, t \in[0, T] .
\end{aligned}
$$

Thus, the equation (1) is equivalent to a singularly perturbed problem for an integro-differential equation with a rapidly oscillating inhomogeneity. Research of such problems was carried out by the method of regularization [1-3], splitting methods [4-6] (for ordinary differential equations). Singularly perturbed integro-differential equations with rapidly oscillating coefficients were studied in [7-13]. Further the function $\lambda_{2}(t)=+i \beta^{\prime}(t)$ will be called the spectrum of a rapidly oscillating inhomogeneity, and the set of functions $\left\{\lambda_{1}(t), \lambda_{2}(t)\right\}$ will be called the spectrum of the problem (1). We will consider problem (3) under the following assumptions:

$$
\begin{aligned}
& \text { 1) } \beta(t), h_{1}(t), h_{2}(t) \in C^{\infty}\left([0, T], \mathbf{R}^{1}\right) \\
& K(t, s) \in C^{\infty}\left(0 \leq s \leq t \leq T, \mathbf{R}^{1} ;\right. \\
& \text { 2) } \lambda_{1}(t) \equiv K(t, t)<0, \beta^{\prime}(t)>0, \forall t \in[0, T] .
\end{aligned}
$$

Let us turn to the development of an algorithm that allows one to construct regularized $[1,2]$ asymptotic solutions of the integro-differential problem (3) (and hence for the original equation (1)).

\section{Regularization of the problem (3) and construction of an extended problem}

Let us introduce regularizing variables:

$$
\tau_{i}=\frac{1}{\varepsilon} \int_{0}^{t} \lambda_{i}(s) d s \equiv \frac{\psi_{i}(t)}{\varepsilon}, \quad i=\overline{1,2}
$$

over the spectrum $\left\{\lambda_{j}(t)\right\}$ and instead of the problem (3) we will consider the following problem for a function $\tilde{y}(t, \tau, \varepsilon)$ of a larger number of variables: 


$$
\begin{aligned}
& L_{\varepsilon} \tilde{y}(t, \tau, \varepsilon) \equiv \varepsilon \frac{\partial \tilde{y}}{\partial t}+\sum_{j=1}^{2} \lambda_{j}(t) \frac{\partial \tilde{y}}{\partial \tau_{j}}-\lambda_{1}(t) \tilde{y} \\
& -\int_{0}^{t} G(t, s) \tilde{y}\left(s, \frac{\psi(s)}{\varepsilon}, \varepsilon\right) d s=h_{1}^{\prime}(t)+\varepsilon h^{\prime}{ }_{2}(t) e^{\tau_{2}} \\
& +i \beta^{\prime}(t) h_{2}(t) e^{\tau_{2}}, \tilde{y}(0,0, \varepsilon)=\frac{h_{1}(0)}{\varepsilon}+h_{2}(0) e^{\frac{i \beta(0)}{\varepsilon}}
\end{aligned}
$$

where $\tau=\left(\tau_{1}, \tau_{2}\right), \psi=\left(\psi_{1}, \psi_{2}\right)$, and $\tau_{1}, \tau_{2}, \tau_{3}$ along side $t$ are independent variables. It is clear that if $y=\tilde{y}(t, \psi(t) / \varepsilon, \varepsilon)$ is the solution to the problem (4), then the vector function $y=\tilde{y}(t, \psi(t) / \varepsilon, \varepsilon)$ is an exact solution to problem (3); therefore, the problem (4) is extended with respect to problem (3). However, it cannot be considered fully regularized, since the integral term

$$
J\left(\left.\tilde{y}(t, \tau, \varepsilon)\right|_{t=s, \tau=\psi(s) / \varepsilon}\right)=\int_{0}^{t} G(t, s) \tilde{y}\left(s, \frac{\psi(s)}{\varepsilon}, \varepsilon\right) d s
$$

has not been regularized in it. To regularize the integral operator, we introduce a class $M_{\varepsilon}$ that is asymptotically invariant with respect to the operator $J$ (see [1], p. 62). Let us recall the corresponding concept.

Definition 1. A class $M_{\varepsilon}$ is said to be asymptotically invariant (for $\varepsilon \rightarrow+0)$ with respect to an operator $P_{0}$ if the following conditions are satisfied:

1) $M_{\varepsilon} \subset D\left(P_{0}\right)$ for each fixed $\varepsilon \neq 0\left(D\left(P_{0}\right)\right.$ is the area of definition of the operator $\left.P_{0}\right)$;

2) The image $P_{0} g(t, \varepsilon)$ of any element $g(t, \varepsilon) \in M_{\varepsilon}$ is expanded in a series

$$
P_{0} g(t, \varepsilon)=\sum_{n=0}^{\infty} \varepsilon^{n} g_{n}(t, \varepsilon)
$$

converging asymptotically as $\varepsilon \rightarrow+0$ (uniformly for $t \in[0, T]$ ).

As a space $U$, we take the space of functions $y(t, \tau)$ representable by the sums

$$
\begin{aligned}
& y(t, \tau)=y_{0}(t)+\sum_{j=1}^{2} y_{i}(t) e^{\tau_{i}}, y_{j}(t) \in C^{\infty}\left([0, T], \mathbf{C}^{1}\right), \\
& j=0,1,2 .
\end{aligned}
$$

Let us show first that the class $M_{\varepsilon}=\left.U\right|_{\tau=\psi(t) / \varepsilon}$ is asymptotically invariant under the operator $J$. The image of the operator $J$ on the element (5) of the 
space $U$ has the form

$$
\begin{aligned}
& J\left(\left.y(t, \tau)\right|_{t=s, \tau=\psi(s) / \varepsilon}\right)=\int_{0}^{t} G(t, s) y\left(s, \frac{\psi(s)}{\varepsilon}\right) d s \\
= & \int_{0}^{t} G(t, s) y_{0}(s) d s+\sum_{j=1}^{2} \int_{0}^{t} G(t, s) y_{j}(s) e^{\frac{1}{\varepsilon} \int_{0}^{s} \lambda_{j}(\theta) d \theta} .
\end{aligned}
$$

Using the operation of integration by parts, we obtain the following expansion

$$
\begin{gathered}
J_{j}(t, \varepsilon)=\sum_{v=0}^{\infty}(-1)^{v} \varepsilon^{\nu+1}\left[\left(I_{j}^{v}\left(G(t, s) y_{j}(s)\right)\right)_{s=t} e^{\frac{1}{\varepsilon} \int_{0}^{t} \lambda_{j}(\theta) d \theta}\right. \\
\left.-\left(I_{j}^{v}\left(G(t, s) y_{j}(s)\right)\right)_{s=0}\right],
\end{gathered}
$$

where $I_{j}^{0}=\frac{1}{\lambda_{j}(s)}, I_{j}^{v}=\frac{1}{\lambda_{j}(s)} \frac{\partial}{\partial s} I_{j}^{v-1}(\nu \geq 1, j=\overline{1,2})$. Therefore, the image of the operator $J$ on an element (5) of the space $U$ is represented as a series

$$
\begin{gathered}
J\left(\left.y(t, \tau)\right|_{t=s, \tau=\psi(s) / \varepsilon}\right)=\int_{0}^{t} G(t, s) y_{0}(s) d s \\
+\sum_{j=1}^{2} \sum_{\nu=0}^{\infty}(-1)^{\nu} \varepsilon^{\nu+1}\left[\left(I_{j}^{v}\left(G(t, s) y_{j}(s)\right)\right)_{s=t} e^{\frac{1}{\varepsilon} \int_{0}^{t}\left(\lambda_{j}(\theta)\right) d \theta}\right. \\
\left.-\left(I_{j}^{v}\left(G(t, s) y_{j}(s)\right)\right)_{s=0}\right] .
\end{gathered}
$$

It is easy to show (see, for example, [14], pp. 291-294) that this series converges asymptotically for $\varepsilon \rightarrow+0$ (uniformly in $t \in[0, T]$ ). This means that the class $M_{\varepsilon}$ is asymptotically invariant (for $\varepsilon \rightarrow+0$ ) with respect to the operator $J$. We introduce operators $R_{v}: U \rightarrow U$ acting on each element $y(t, \tau, \sigma) \in U$ of the form (5) according to the law:

$$
\begin{gathered}
R_{0} y(t, \tau)=\int_{0}^{t} G(t, s) y_{0}(s) d s \\
R_{1} y(t, \tau)=\sum_{j=1}^{2}\left[\left(I_{j}^{0}\left(G(t, s) y_{j}(s)\right)\right)_{s=t} e^{\tau_{j}}\right. \\
\left.-\left(I_{j}^{0}\left(G(t, s) y_{j}(s)\right)\right)_{s=0}\right], \\
R_{v+1} y(t, \tau)=\sum_{j=1}^{2}(-1)^{v} \varepsilon^{v+1}\left[\left(I_{j}^{v}\left(G(t, s) y_{j}(s)\right)\right)_{s=t} e^{\tau_{j}}\right. \\
\left.-\left(I_{j}^{v}\left(G(t, s) y_{j}(s)\right)\right)_{s=0}\right] .
\end{gathered}
$$


Operators $R_{v}: U \rightarrow U$ are called order operators because they extract in an expression $J y(t, \tau, \sigma)$ the sum of order $\nu$ relative to a parameter $\varepsilon$.

Now let $\tilde{y}(t, \tau, \varepsilon)$ be an arbitrary continuous function in $(t, \tau) \in[0, T] \times$ $\left\{\tau: \operatorname{Re} \tau_{j} \leq 0, j=\overline{1,2}\right\}$ with an asymptotic expansion

$$
\tilde{y}(t, \tau, \varepsilon)=\sum_{k=-1}^{\infty} \varepsilon^{k} y_{k}(t, \tau), y_{k}(t, \tau) \in U
$$

converging (when $\varepsilon \rightarrow+0$ ) uniformly in

$$
(t, \tau) \in[0, T] \times\left\{\tau: \operatorname{Re} \tau_{j} \leq 0, j=\overline{1,2}\right\}
$$

This means that for any fixed $N=-1,0,1, \ldots$, there is a constant $C_{N}>0$ independent of $\varepsilon, t, \tau$ such that the inequality

$$
\left\|\tilde{y}(t, \tau, \varepsilon)-\sum_{k=-1}^{N} \varepsilon^{k} y_{k}(t, \tau)\right\|_{C[0, T]} \leq C_{N} \varepsilon^{N+1}
$$

holds true for $0<\left|\varepsilon-\varepsilon_{0}\right|<\delta_{N}$, where $\delta_{N}>0$ is small enough value. Then the image $J \tilde{y}$ of this function expands into an asymptotic series

$$
\begin{aligned}
& J\left(\left.\tilde{y}(t, \tau, \varepsilon)\right|_{\tau=\psi(t) / \varepsilon}\right)=\sum_{k=-1}^{\infty} \varepsilon^{k} J\left(\left.y_{k}(t, \tau)\right|_{\tau=\psi(t) / \varepsilon}\right) \\
& =\left.\sum_{r=-1}^{\infty} \varepsilon^{r} \sum_{s=-1, r-s \geq 0}^{r} R_{r-s} y_{s}(t, \tau)\right|_{\tau=\psi(t) / \varepsilon} .
\end{aligned}
$$

This equality is the basis for introducing an extension of the operator $J$ on series of the form (7):

$$
\begin{gathered}
\tilde{J} \tilde{y}(t, \tau, \varepsilon) \\
\equiv \tilde{J}\left(\sum_{k=-1}^{\infty} \varepsilon^{k} y_{k}(t, \tau)\right)=\sum_{r=-1}^{\infty} \varepsilon^{r} \sum_{s=-1, r-s \geq 0}^{r} R_{r-s} y_{s}(t, \tau) .
\end{gathered}
$$

Although the operator $\tilde{J}$ is defined formally, its usefulness is obvious, since in practice one usually constructs an $N$-th approximation of the asymptotic solution of the equation (3), in which only $N$-th partial sums of the series (7) will participate, which have not a formal but a true meaning. Now we can write down the problem, which is completely regularized with respect to the original equation (2): 


$$
\begin{aligned}
& L_{\varepsilon} \tilde{y}(t, \tau, \varepsilon) \equiv \varepsilon \frac{\partial \tilde{y}}{\partial t}+\sum_{j=1}^{2} \lambda_{j}(t) \frac{\partial \tilde{y}}{\partial \tau_{j}}-\lambda_{1}(t) \tilde{y} \\
& -\tilde{J} \tilde{y}(t, \tau, \varepsilon)=h_{1}^{\prime}(t)+\varepsilon h_{2}^{\prime}(t) e^{\tau_{2}}+i \beta^{\prime}(t) h_{2}(t) e^{\tau_{2}}, \\
& \tilde{y}(0,0, \varepsilon)=\frac{h_{1}(0)}{\varepsilon}+h_{2}(0) e^{\frac{i \beta(0)}{\varepsilon}}, \quad t \in[0, T] .
\end{aligned}
$$

\section{Iterative problems and their solvability in space $U$. Remainder estimate theorem}

We will define the solution to the problem (8) as a series (7). After equating the coefficients at the same degrees $\varepsilon$ (taking into account the formulas $\left.\left(6_{0}\right),\left(6_{1}\right), \ldots,\left(6_{v}\right)\right)$, we obtain the following iterative problems:

$$
\begin{aligned}
& L_{0} y_{-1}(t, \tau) \equiv \sum_{i=1}^{2} \lambda_{i}(t) \frac{\partial_{y-1}}{\partial \tau_{i}}-\lambda_{1}(t) y_{-1} \\
& -\int_{0}^{t} G(t, s) y_{0}^{(-1)}(s) d s=0, \quad y_{-1}(0,0)=h_{1}(0) ; \\
& L_{0} y_{0}(t, \tau)=-\frac{\partial y_{-1}}{\partial t}+R_{1} y_{-1}+h^{\prime}{ }_{1}(t)+i \beta^{\prime}(t) h_{2}(t) e^{\tau_{2}}, \\
& y_{0}(0,0)=h_{2}(0) e^{\frac{i \beta(0)}{\varepsilon}} \\
& L_{0} y_{1}(t, \tau)=-\frac{\partial y_{k-1}}{\partial t}+R_{1} y_{0}+R_{2} y_{-1}, \quad y_{1}(0,0)=0 ; \\
& L_{0} y_{k}(t, \tau)=-\frac{\partial y_{k-1}}{\partial t}+R_{1} y_{k-1}+\ldots+R_{k+1} y_{-1}, \\
& y_{k}(0,0)=0, \quad k \geq 0 .
\end{aligned}
$$

Each of the iterative problems $\left(9_{k}\right)$ has the form

$$
\begin{aligned}
& L_{0} y(t, \tau) \equiv \sum_{j=1}^{2} \lambda_{j}(t) \frac{\partial y}{\partial \tau_{j}}-\lambda_{1}(t) y \\
& -\int_{0}^{t} G(t, s) y_{0}(s) d s=H(t, \tau), \quad y(0,0)=y_{*},
\end{aligned}
$$

where $y_{*}$ is a known number, $H(t, \tau)$ is a known function of a class $U$ whose elements are the sums 


$$
\begin{aligned}
& H(t, \tau)=H_{0}(t)+\sum_{j=1}^{2} H_{j}(t) e^{\tau_{j}}, \\
& H_{j}(t) \in C^{\infty}\left([0, T], \mathbf{C}^{1}\right), \quad j=\overline{0,2} .
\end{aligned}
$$

We introduce the scalar (for each $t \in[0, T]$ ) product in the space $U$ :

$$
\begin{gathered}
<z, w>\equiv<z_{0}(t)+\sum_{j=1}^{2} z_{j}(t) e^{\tau_{j}}, w_{0}(t)+\sum_{j=1}^{2} w_{j}(t) e^{\tau_{j}}> \\
=z_{0}(t) \overline{w_{0}(t)}+\sum_{j=1}^{2} z_{j}(t) \overline{w_{j}(t)},
\end{gathered}
$$

where the bar above the function $w(t)$ means complex conjugation in $\mathbf{C}^{1}$. Let us prove the following statement.

Theorem 1. Let conditions 1) and 2) be satisfied and the right-hand side $H(t, \tau)=H_{0}(t)+\sum_{j=1}^{2} H_{j}(t) e^{\tau_{j}}$ of the equation (10) belongs to the space $U$. Then, for the solvability of the system (10) in $U$ it is necessary and sufficient that the identity

$$
<H(t, \tau), e^{\tau_{1}}>\equiv 0, \forall t \in[0, T]
$$

is carried out.

Proof. We will define the solution of the equation (10) in the form of an element (5) of space $U$ :

$$
z(t, \tau)=z_{0}(t)+\sum_{j=1}^{2} z_{j}(t) e^{\tau_{j}} .
$$

Substituting (12) into the equation (10), we will have

$$
\begin{gathered}
\sum_{j=1}^{2}\left[\lambda_{j}(t)-\lambda_{1}(t)\right] z_{i}(t) e^{\tau_{i}}-\lambda_{1}(t) z_{0}(t)-\int_{0}^{t} G(t, s) z_{0}(s) d s \\
=H_{0}(t)+\sum_{j=1}^{2} H_{j}(t) e^{\tau_{j}}
\end{gathered}
$$

Equating here separately the free terms and coefficients at the same exponents, we obtain the following equations:

$$
\begin{gathered}
-\lambda_{1}(t) z_{0}(t)-\int_{0}^{t} G(t, s) z_{0}(s) d s=H_{0}(t), \\
{\left[\lambda_{j}(t)-\lambda_{1}(t)\right] z_{j}(t)=H_{j}(t), \quad j=\overline{1,2} .}
\end{gathered}
$$


Since the function $\lambda_{1}(t)<0(\forall t \in[0, T])$, then the equation (14) can be written in the form

$$
z_{0}(t)=\int_{0}^{t}\left(-\lambda_{1}^{-1}(t) G(t, s)\right) z_{0}(s) d s-\lambda_{1}^{-1}(t) H_{0}(t) .
$$

Due to the smoothness of the kernel $-\lambda_{1}^{-1}(t) K(t, s)$ and the inhomogeneity $-\lambda_{1}^{-1}(t) H_{0}(t)$, this Volterra integral equation has a unique solution $z_{0}(t) \in$ $C^{\infty}\left([0, T], \mathbf{C}^{1}\right)$ The equation $\left(14_{2}\right)$ also has a unique solution

$$
z_{2}(t)=\left[\lambda_{2}(t)-\lambda_{1}(t)\right]^{-1} \in C^{\infty}\left([0, T], \mathbf{C}^{1}\right),
$$

since $\lambda_{2}(t)$ does not coincide with $\lambda_{1}(t)$ for all $t \in[0, T]$. The equation $\left(14_{1}\right)$ is solvable in the space $C^{\infty}\left([0, T], \mathbf{C}^{1}\right)$ if and only if the identity

$$
H_{1}(t) \equiv 0 \Leftrightarrow\left\langle H_{1}(t), e^{\tau_{1}}\right\rangle \equiv 0 \quad \forall t \in[0, T]
$$

holds. Thus, condition (11) is necessary and sufficient for the solvability of the equation (10) in the space $U$. The theorem is proved.

Remark 1. If identity (11) holds, then under conditions 1) and 2) the equation (10) has the following solution in the space $U$ :

$$
z(t, \tau)=z_{0}(t)+\alpha_{1}(t) e^{\tau_{1}}+\frac{H_{2}(t)}{\lambda_{2}(t)-\lambda_{1}(t)} e^{\tau_{2}},
$$

where $\alpha_{1}(t) \in C^{\infty}\left([0, T], \mathbf{C}^{1}\right)$ is an arbitrary function, $z_{0}(t)$ is the solution of the integral equation $\left(15_{0}\right)$.

We will not formulate a theorem on the unique solvability of the problem (10). Let us show that the application of Theorem 1 to two sequential iterative problems $\left(9_{k}\right)$ and $\left(9_{k+1}\right)$ allows us to determine the solution of the first of them uniquely in the class $U$. Let's start with the first iterative $\left(9_{-1}\right)$. The righthand side $H(t, \tau)=H^{(-1)}(t, \tau) \equiv 0$ of this problem satisfies the solvability condition (11); therefore, the equation $\left(9_{-1}\right)$ has a solution in the space $U$ in the form of a function (see (16))

$$
y_{-1}(t, \tau)=y_{0}^{(-1)}(t)+\alpha_{1}^{(-1)}(t) e^{\tau_{1}},
$$

where the function $y_{0}^{(-1)}(t)$ satisfies the homogeneous integral equation

$$
y_{0}^{(-1)}(t)=\int_{0}^{t}\left(-\lambda_{1}^{-1}(t) G(t, s)\right) y_{0}^{(-1)}(s) d s
$$


which has a unique zero solution $y_{0}^{(-1)}(t) \equiv 0$. Hence, $y_{-1}(t, \tau)=\alpha_{1}^{(-1)}(t) e^{\tau_{1}}$, where $\alpha_{1}^{(-1)}(t) \in C^{\infty}\left([0, T], \mathbf{C}^{1}\right)$ is still an arbitrary function. Submitting it to the initial condition $y_{-1}(0,0)=h_{1}(0)$, we find the value $\alpha_{1}^{(-1)}(0)=h_{1}(0)$. For the final calculation of the function $\alpha_{1}^{(-1)}(t)$, we pass to the following iterative problem:

$$
\begin{aligned}
& L_{0} y_{0}=-\frac{\partial y_{-1}}{\partial t}+R_{1} y_{-1}+h_{1}^{\prime}(t)+i \beta^{\prime}(t) h_{2}(t) e^{\tau_{2}}, \\
& y_{0}(0,0)=h_{2}(0) e^{\frac{i \beta(0)}{\varepsilon}} .
\end{aligned}
$$

Taking into account the form of the operator $R_{1}$, we write down the right-hand side of problem (17):

$$
\begin{aligned}
& H(t, \tau)=H^{(0)}(t, \tau) \equiv-\dot{\alpha}_{1}^{(-1)}(t) e^{\tau_{1}} \\
& +R_{1}\left(\alpha_{1}^{(-1)}(t) e^{\tau_{1}}\right)+h^{\prime}{ }_{1}(t)+i \beta^{\prime}(t) h_{2}(t) e^{\tau_{2}} \\
& =-\dot{\alpha}_{1}^{(-1)}(t) e^{\tau_{1}}+\frac{G(t, t) \alpha_{1}^{(-1)}(t)}{\lambda_{1}(t)} e^{\tau_{1}} \\
& -\frac{G(t, 0) \alpha_{1}^{(-1)}(0)}{\lambda_{1}(0)}+h^{\prime}{ }_{1}(t)+i \beta^{\prime}(t) h_{2}(t) e^{\tau_{2}} .
\end{aligned}
$$

The solvability condition (11) of this equation in the space $U$ leads to the differential equation $-\dot{\alpha}_{1}^{(-1)}(t)+\frac{G(t, t)}{\lambda_{1}(t)} \alpha_{1}^{(-1)}(t)=0$. Submitting it to the initial condition $\alpha_{1}^{(-1)}(0)=h_{1}(0)$, we will uniquely find the function $\alpha_{1}^{(-1)}(t)=h_{1}(0) e^{\int_{0}^{t} \frac{G(\theta, \theta) d \theta}{\lambda_{1}(\theta)}}$ and calculate the solution

$$
y_{-1}(t, \tau)=h_{1}(0) e^{\int_{0}^{t} \frac{G(\theta, \theta) d \theta}{\lambda_{1}(\theta)}+\tau_{1}}
$$

of the problem $\left(9_{-1}\right)$ in the space $U$ uniquely. In this case, the problem $\left(9_{0}\right)$ takes the form

$$
\begin{gathered}
L_{0} y_{0}=-\frac{G(t, 0) h_{1}(0)}{\lambda_{1}(0)}+h_{1}^{\prime}(t)+i \beta^{\prime}(t) h_{2}(t) e^{\tau_{2}}, \\
y_{0}(0,0)=h_{2}(0) e^{\frac{i \beta(0)}{\varepsilon}} .
\end{gathered}
$$

By Theorem 1 (see formula (16)), it has the following solution in the space $U$ :

$$
y_{0}(t, \tau)=y_{0}^{(0)}(t)+\alpha_{1}^{(0)}(t) e^{\tau_{1}}+\frac{i \beta^{\prime}(t) h_{2}(t)}{\lambda_{2}(t)-\lambda_{1}(t)} e^{\tau_{2}},
$$


where $\alpha_{1}^{(0)}(t) \in C^{\infty}\left([0, T], \mathbf{C}^{1}\right)$ is an arbitrary function and $y_{0}^{(0)}(t)$ is the solution to the integral equation

$$
\begin{aligned}
& y_{0}^{(0)}(t)=\int_{0}^{t}\left(-\frac{G(t, s)}{\lambda_{1}(t)}\right) y_{0}^{(0)}(s) d s \\
& -\frac{1}{\lambda_{1}(t)}\left(-\frac{G(t, 0) h_{1}(0)}{\lambda_{1}(0)}+h_{1}^{\prime}(t)\right) .
\end{aligned}
$$

Submitting the solution (19) to the initial condition $y_{0}(0,0)=h_{2}(0) e^{\frac{i \beta(0)}{\varepsilon}}$, we find the initial value

$$
\begin{aligned}
& \alpha_{1}^{(0)}(0)=\frac{1}{\lambda_{1}(0)}\left(-\frac{G(t, 0) h_{1}(0)}{\lambda_{1}(0)}+h_{1}^{\prime}(0)\right) \\
& -\frac{i \beta^{\prime}(0) h_{2}(0)}{\lambda_{2}(0)-\lambda_{1}(0)}+h_{2}(0) e^{\frac{i \beta(0)}{\varepsilon}} \equiv \alpha_{1}^{0} .
\end{aligned}
$$

For a complete calculation of the function $\alpha_{1}^{(0)}(t)$, it is necessary to go to the next iterative problem $\left(9_{1}\right)$ and write down the solvability conditions (11) for it. They will lead to the inhomogeneous differential equation

$$
\begin{aligned}
& -\dot{\alpha}_{1}^{(0)}(t)+\frac{G(t, t)}{\lambda_{1}(t)} \alpha_{1}^{(0)}(t)=l(t), \alpha_{1}^{(0)}(0)=\alpha_{1}^{0}, \\
& l(t) \equiv \frac{1}{\lambda_{1}(t)}\left(\frac{\partial}{\partial s}\left(\frac{G(t, s)}{\lambda_{1}(s)} h_{1}(0) e^{\int} \frac{G(\theta, \theta) d \theta}{\lambda_{1}(\theta)}\right)\right)_{s=t} .
\end{aligned}
$$

This equation is satisfied by a single function:

$$
\alpha_{1}^{(0)}(t)=\alpha_{1}^{0} e^{\int^{t} \frac{G(\theta, \theta) d \theta}{\lambda_{1}(\theta)}}+\int_{0}^{t} e^{-\int_{0}^{s} \frac{G(\theta, \theta) d \theta}{\lambda_{1}(\theta)}} l(s) d s
$$

and, therefore, the solution (19) of the problem $\left(9_{0}\right)$ will be calculated uniquely in the space $U$. And similarly, applying Theorem 1 to the series of the following iterative problems $\left(9_{k}\right)$, we will uniquely find their solutions in the space $U$ and construct the series (7). Just as in [16], we prove the following statement.

Theorem 2. Let conditions 1) - 2) be satisfied for equation (3). Then, for $\varepsilon \in\left(0, \varepsilon_{0}\right]$ (where $\varepsilon_{0}>0$ is sufficiently small), the equation (3) has a unique solution $y(t, \varepsilon) \in C^{1}([0, T], \mathbf{C})$; in this case, the estimate

$$
\left\|y(t, \varepsilon)-y_{\varepsilon N}(t)\right\|_{C[0, T]} \leq c_{N} \varepsilon^{N+1}, \quad N=-1,0,1, \ldots
$$


takes place. Here $y_{\varepsilon N}(t)$ is the restriction (at $\tau=\frac{\psi(t)}{\varepsilon}$ ) of the $N$-th partial sum of series (7) (with coefficients $y_{k}(t, \tau) \in U$, satisfying the iterative problems $\left.\left(9_{k}\right)\right)$, and the constant $c_{N}>0$ does not depend on $\varepsilon$ at $\varepsilon \in\left(0, \varepsilon_{0}\right]$.

Let us write down the leading term of the asymptotics of the solution to problem (3) (and hence to the original problem (1)):

$$
\begin{aligned}
& y_{\varepsilon 0}(t)=\varepsilon^{-1} y_{-1}\left(t, \frac{\psi(t)}{\varepsilon}\right)+y_{0}\left(t, \frac{\psi(t)}{\varepsilon}\right) \\
& =\varepsilon^{-1} h_{1}(0) e^{\int^{t} \frac{G(\theta, \theta) d \theta}{\lambda_{1}(\theta)}+\frac{1}{\varepsilon} \int_{0}^{t} \lambda_{1}(\theta) d \theta}+y_{0}^{(0)}(t) \\
& +\left(\alpha_{1}^{0} e^{\int^{t} \frac{G(\theta, \theta) d \theta}{\lambda_{1}(\theta)}}+\int_{0}^{t} e^{-\int_{0}^{s} \frac{G(\theta, \theta) d \theta}{\lambda_{1}(\theta)}} l(s) d s\right) e^{\frac{1}{\varepsilon} \int_{0}^{t} \lambda_{1}(\theta) d \theta} \\
& +\frac{i \beta^{\prime}(t) h_{2}(t)}{\lambda_{2}(t)-\lambda_{1}(t)} e^{\frac{1}{\varepsilon} \int_{0}^{t} \lambda_{2}(\theta) d \theta}
\end{aligned}
$$

(where $l(t)$ is calculated by formula $(20 \mathrm{a})$ ) and analyze it. If $h_{1}(0) \neq 0$, then the exact solution $y(t, \varepsilon)$ of the problem (1), tending to infinity when $\varepsilon \rightarrow+0$ at the point $t=0$, performs at $t>0$ fast oscillations around $y_{0}^{(0)}(t)$. If $h_{1}(0)=0$, then the solution $y(t, \varepsilon)$, remaining bounded when $\varepsilon \rightarrow+0$, performs at $t>0$ fast oscillations around the solution $y_{0}^{(0)}(t)$ of the integral equation (20). It is easy to see that this equation is obtained from a degenerate (with respect to (1)) equation with a jump:

$$
-\int_{0}^{t} K(t, s) \overline{\bar{y}}(s) d s=h_{1}(t)+\Delta(t),
$$

where $\Delta(t)=-\int_{0}^{t} \frac{G(x, 0)}{\lambda_{1}(0)} h_{1}(0) d x$.

Remark 2. We note the following interesting fact: the solution $y_{0}^{(0)}(t)$ cannot be obtained from the integro-differential equation (2), putting $\varepsilon=0$ in it, since the term $i \beta^{\prime}(t) h_{2}(t) e^{\frac{i \beta(t)}{\varepsilon}}$ on the right-hand side of the equation (2) is not defined at $\varepsilon=0$. This fact is confirmed by an example of the following integral equation:

$$
\varepsilon y(t)=-\int_{0}^{t} y(x) d x+\varepsilon \cdot e^{\frac{i \cdot t}{\varepsilon}}
$$

whose solution is the function

$$
y(t, \varepsilon)=\left(\frac{1}{2}+\frac{1}{2} i\right) e^{\frac{i t}{\varepsilon}}+\left(\frac{1}{2}-\frac{1}{2} i\right) e^{-\frac{t}{\varepsilon}} .
$$


The degenerate solution (at $\varepsilon=0$; more precisely, when $\varepsilon \rightarrow+0$ ) of the integral equation (21) is the identical zero $\overline{\bar{y}}(t) \equiv 0$. When $\varepsilon \rightarrow+0$, the exact solution $y(t, \varepsilon)$ of the equation (21), while remaining bounded, performs fast oscillations at $t>0$ near $\overline{\bar{y}}(t) \equiv 0$. If, however, we differentiate the integral equation (21) by $t$, we obtain the integro-differential problem

$$
\varepsilon \frac{d}{d t} y(t)=-y(t)+i \cdot e^{\frac{i \cdot t}{\varepsilon}}, \quad y(0)=1
$$

whose solution is also function (22). However, it is impossible to put $\varepsilon=0$ in the problem (23), since the expression $i \cdot e^{\frac{i \cdot t}{\varepsilon}}$ for $\varepsilon=0$ is meaningless.

Example. Let the equation be given

$$
\begin{aligned}
\varepsilon y(t)= & -\int_{0}^{t}(t-s+1) y(s) d s+t^{2}+1 \\
& +\varepsilon(t+1) \cdot e^{\frac{i \cdot t}{\varepsilon}}, \quad t \in[0, T] .
\end{aligned}
$$

It is possible to obtain its solution in quadratures:

$$
\begin{gathered}
y(t)=-\frac{1}{2} e^{1 / 2 \frac{(-1+\sqrt{1-4 \varepsilon}) t}{\varepsilon}}\left(2 i \sqrt{1-4 \varepsilon} \varepsilon^{3}+2 i \sqrt{1-4 \varepsilon \varepsilon^{2}-4 i \varepsilon^{3}}\right. \\
+\sqrt{1-4 \varepsilon} \varepsilon^{3}-2 \varepsilon^{4}-5 i \sqrt{1-4 \varepsilon} \varepsilon+8 i \varepsilon^{2}-5 \sqrt{1-4 \varepsilon} \varepsilon^{2} \\
+\varepsilon^{3}+2 i \sqrt{1-4 \varepsilon}-3 i \varepsilon \\
\left.+3 \sqrt{1-4 \varepsilon \varepsilon}-\varepsilon^{2}-2 i-3 \varepsilon\right)(\sqrt{1-4 \varepsilon})^{-1} \varepsilon^{-1}(2 i \varepsilon \\
\left.+\varepsilon^{2}-2 i-2 \varepsilon\right)^{-1}-\frac{1}{2} \mathrm{e}^{-1 / 2 \frac{(1+\sqrt{1-4 \varepsilon}) t}{\varepsilon}}\left(2 i \sqrt{1-4 \varepsilon} \varepsilon^{3}+2 i \sqrt{1-4 \varepsilon} \varepsilon^{2}\right. \\
+4 i \varepsilon^{3}+\sqrt{1-4 \varepsilon} \varepsilon^{3} \\
+2 \varepsilon^{4}-5 i \sqrt{1-4 \varepsilon \varepsilon}-8 i \varepsilon^{2}-5 \sqrt{1-4 \varepsilon} \varepsilon^{2}-\varepsilon^{3}+2 i \sqrt{1-4 \varepsilon} \\
+3 i \varepsilon+3 \sqrt{1-4 \varepsilon} \varepsilon+\varepsilon^{2}+2 i \\
+3 \varepsilon)(\sqrt{1-4 \varepsilon})^{-1} \varepsilon^{-1}\left(2 i \varepsilon+\varepsilon^{2}-2 i-2 \varepsilon\right)^{-1}+\left(\left(2 i \varepsilon^{2}\right.\right. \\
+(-t-2) \varepsilon+1-i+(1-i) t) \mathrm{e}^{\frac{i t}{\varepsilon}}-4 i \\
\left.+2 \varepsilon^{2}-(4-4 i) \varepsilon\right)\left(-2 i+\varepsilon^{2}-(2-2 i) \varepsilon\right)^{-1}
\end{gathered}
$$

(for example, using the Maple program), but it will hardly be possible to isolate the asymptotic solution of equation (24) in it. We will do this using the 
algorithm of the regularization method developed by us. Let us use the formula $(*)$. In our case

$$
\begin{aligned}
& K(t, s)=-(t-s+1), \lambda_{1}(t)=K(t, t)=-1, \\
& G(t, s)=-1, \quad h_{1}(t)=t^{2}+1, h_{2}(t)=t+1, \\
& \beta(t)=t, \quad \lambda_{2}(t)=i \beta^{\prime}(t)=i \\
& \alpha_{1}^{(-1)}(0)=h_{1}(0)=1 .
\end{aligned}
$$

The function $y_{0}^{(0)}(t)$ is the solution to the integral equation

$$
\begin{aligned}
y_{0}^{(0)}(t) & =\int_{0}^{t}\left(-\frac{G(t, s)}{\lambda_{1}(t)}\right) y_{0}^{(0)}(s) d s-\frac{h_{1}^{\prime}(t)}{\lambda_{1}(t)} \Leftrightarrow \\
& \Leftrightarrow y_{0}^{(0)}(t)=-\int_{0}^{t} y_{0}^{(0)}(s) d s+2 t
\end{aligned}
$$

which is easily solved by differentiating on $t$ :

$$
\begin{aligned}
\frac{d y_{0}^{(0)}(t)}{d t} & =-y_{0}^{(0)}(t)+2, y_{0}^{(0)}(0)=0 \Leftrightarrow \\
& \Leftrightarrow y_{0}^{(0)}(t)=2-2 e^{-t} .
\end{aligned}
$$

Thus, the leading term of the asymptotics of the solution to equation (1) has the form

$$
\begin{gathered}
y_{\varepsilon 0}(t)=\varepsilon^{-1} y_{-1}\left(t, \frac{\psi(t)}{\varepsilon}\right)+y_{0}\left(t, \frac{\psi(t)}{\varepsilon}\right) \\
=\varepsilon^{-1} h_{1}(0) e^{\int_{0}^{t} \frac{G(\theta, \theta) d \theta}{\lambda_{1}(\theta)}+\frac{1}{\varepsilon} \int_{0}^{t} \lambda_{1}(\theta) d \theta}+y_{0}^{(0)}(t) \\
+\left(\alpha_{1}^{0} e^{\int_{0}^{t} \frac{G(\theta, \theta) d \theta}{\lambda_{1}(\theta)}}+\int_{0}^{t} e^{-\int_{0}^{s} \frac{G(\theta, \theta) d \theta}{\lambda_{1}(\theta)}} l(s) d s\right) e^{\frac{1}{\varepsilon} \int_{0}^{t} \lambda_{1}(\theta) d \theta} \\
+\frac{i \beta^{\prime}(t) h_{2}(t)}{\lambda_{2}(t)-\lambda_{1}(t)} e^{\frac{1}{\varepsilon} \int_{0}^{t} \lambda_{2}(\theta) d \theta} . \\
y_{\varepsilon 0}(t)=\varepsilon^{-1} e^{t-\frac{t}{\varepsilon}}+2-2 e^{-t}+\left(-\frac{i}{i+1}+e^{\frac{i}{\varepsilon}}\right) e^{t} \\
+\left(\int_{0}^{t} e^{-s} l(s) d s\right) e^{-\frac{t}{\varepsilon}}+\frac{i(t+1)}{i+1} e^{-\frac{i t}{\varepsilon}}
\end{gathered}
$$

\section{Acknowledgments}

This work was supported by Grant No. AP05133858 "Contrast structures in singularly perturbed equations and their application in the theory of phase transitions" (2018-2020) of the Committee of Science of the Ministry of Education and Science of the Republic of Kazakhstan. 


\section{References}

[1] S.A. Lomov, Introduction to the General Theory of Singular Perturbations, Nauka, Moscow (1981).

[2] S.A. Lomov, I.S. Lomov, Fundamentals of the Mathematical Theory of Boundary Layer, Moscow University Press, Moscow (2011).

[3] A.D. Ryzhikh, Asymptotic solution of a linear differential equation with a rapidly oscillating coefficient, Trudy Moskow. Energetich. Instituta, 357 (1978), 92-94.

[4] N.I. Shkil, Asymptotic Methods in Differential Equations, Naukova Dumka, Kiev (1971).

[5] S.F. Feshchenko, N.I. Shkil, L.D. Nikolenko, Asymptotic Methods in the Theory of Linear Differential Equations, Naukova Dumka, Kiev (1966).

[6] Yu.L. Daletsky, Asymptotic method for some differential equations with oscillating coefficients, DAN SSSR, 143, No 5 (1962), 1026-1029.

[7] B.T. Kalimbetov, V.F. Safonov, Integro-differentiated singularly perturbed equations with fast oscillating coefficients, Bulletin of KarSU, Ser. Mathematics, 94, No 2 (2019), 33-47.

[8] B.T. Kalimbetov, M.A. Temirbekov, Zh.O. Khabibullaev, Asymptotic solution of singular perturbed problems with an instable spectrum of the limiting operator, Abstr. Appl. Analysis 2012 (2012), Art. ID 120192; doi: $10.1155 / 2012 / 120192$.

[9] A.A. Bobodzhanov, V.F. Safonov, Singularly perturbed nonlinear integrodifferential systems with rapidly varying kernels, Mathematical Notes, $\mathbf{7 2}$, No 5 (2002), 605-614; doi: 10.1023/A:1021444603184.

[10] A.A. Bobodzhanov, V.F. Safonov, Singularly perturbed integrodifferential equations with diagonal degeneration of the kernel in reverse time, Differential Equations, 40, No 1 (2004), 120-127; doi: 10.1023/B:DIEQ.0000028721.81712.67.

[11] A.A. Bobodzhanov, B.T. Kalimbetov, V.F. Safonov, Integro-differential problem about parametric amplification and its asymptotical integration, International Journal of Applied Mathematics, 33, No 2 (2020), 331-353; doi: 10.12732/ijam.v33i2.12. 
[12] B.T. Kalimbetov, V.F. Safonov, Regularization method for singularly perturbed integro-differential equations with rapidly oscillating coefficients and with rapidly changing kernels, Axioms, 9, No 4 (2020), \# 131; doi: 10.3390/axioms9040131.

[13] V.F. Safonov, O.D. Tuychiev, Regularization of singularly perturbed integral equations with rapidly changing kernels, Differ. Uravn., 31, No 9 (1997), 1199-1211.

[14] V.F. Safonov, A.A. Bobodzhanov, Higher Mathematics Course. Singularly Perturbed Problems and the Regularization Method: Uch. Allowance, Publishing House MEI, Moscow (2012). 